\title{
Synthèse de nouveaux dérives de la $R-(+)$-pulégone par la technique de four à micro-onde en milieu sec et étude de leur impact sur les champignons attaquant la pomme dans les entrepôts de stockage
}

\author{
Manuscrit reçu le 7 mai 2015 et accepté le 27 juin 2016 \\ Ahmad OUBAIR $^{1^{*}}$, Rachid FIHI ${ }^{1}$, Hamid MAZOUZ ${ }^{2}$ \\ ${ }^{1}$ Laboratoire des substances naturelles \& synthèse et dynamique moléculaire, Faculté des sciences \\ et techniques Errachidia, Université Moulay Smail, Meknès, Maroc. \\ ${ }^{2}$ Laboratoire de Biotechnologies Végétales \& Biologie Moléculaire, Faculté des Sciences Meknès, \\ Université Moulay Smail, Meknès, Maroc.
}

\section{Résumé}

Dans ce travail on a réalisé la condensation de la R-(+)-pulégone avec une série d'aryaldéhyde en présence d'hydrure de sodium. L'activation par micro-ondes dans des conditions sans solvant, a été applique dans le protocole de synthèse. Le processus est très efficace. Il permet en quelques minutes de préparer de nouveaux dérivés de la pulégone avec des rendements remarquables. Les analyses spectroscopiques à savoir RMN ${ }^{1} \mathrm{H},{ }^{13} \mathrm{C}$ et IR permet d'envisager les structures des nouveaux produits synthétisés. Ainsi on a étudié l'impact de ces nouveaux produits sur la croissance mycélienne des souches des champignons $P$. expansum, Alternariat $s p$ et $R$. stolonifer, isolées des pommes pourries en post-récolte.

Mots clés : R-(+)-pulégone, Arylaldéhyde, Micro-onde, Champignon, Pomme

\begin{abstract}
In this work we performed the condensation of the $\mathrm{R}-(+)$ - pulegone with a series of aryaldehyde in the presence of sodium hydride. The activation by microwaves under conditions without solvent, was applied in the synthesis protocol. The process is very effective. It allows in a few minutes to prepare new derivatives of pulegone with remarkable returns. Spectroscopic analysis of $1 \mathrm{H}$, 13C NMR, and IR allows to consider the structures of the new synthesized products. So we studied the impact of these new products on mycelia growth of fungi strains $P$. expansum, Alternariat $s p$ and $R$. stolonifer, isolated from apple in postharvest.
\end{abstract}

Keywords: R-(+)-pulegone, Arylaldehyde, Microwave, Mushroom, Apple

\section{Introduction}

La R-(+)-pulégone est une énone naturelle obtenue et identifiée à partir des huiles essentielles de diverses plantes [1- 4]. Cette ènone monoterpénique est largement utilisée en industrie pharmaceutique, agroalimentaire et cosmétique ainsi qu'en médecine traditionnelle [5-7]. Plusieurs travaux sont orientés vers l'étude de l'activité biologique des huiles essentielles extraites de différentes plantes dont le constituant majoritaire est la R-(+)-pulégone [8-26], tandis que d'autres ont utilisé la R-(+)-pulégone comme intermédiaire

\footnotetext{
* Auteur Correspondent. E-mail : oubair_hmad@yahoo.fr
} 
dans la synthèse d'un bon nombre de produits [27-43].

Dans le cadre des travaux de recherche de notre équipe sur la valorisation des substances naturelles [44-48], nous nous sommes intéressés à la synthèse de nouveaux dérivés de la R-(+)-pulégone. Ces produits peuvent être utilisés comme synthons dans la synthèse des composés à valeurs ajoutées, mais l'activation des réactions de synthèse organique en général, demeure une préoccupation fondamentale, puisque l'optimisation des rendements et le respect de l'environnement par la contribution au fardeau de la pollution chimique, sont liés au mode d'activation choisi. À cet égard, on a recours à la technique du four à micro-ondes qui est utilisée d'après la littérature dans la synthèse d'une grande variété de composés industriellement importants et des produits intermédiaires, à savoir les énones, les hétérocycles ainsi que dans différentes extractions [49-50].

Cette approche synthétique repose sur la condensation de la R-(+)-pulégone avec les arylaldéhydes en présence de l'hydrure de sodium sous l'irradiation micro-ondes en milieu sec. Les nouveaux composés ont été caractérisés par différentes méthodes spectroscopique à savoir IR, RMN ${ }^{1} \mathrm{H}$ et $\mathrm{RMN}{ }^{13} \mathrm{C}$.

Afin de valoriser les produits synthétisés, une étude biologique a été réalisée. Elle consiste à évaluer le pouvoir inhibiteur de chaque produit sur la croissance mycélienne de trois souches de champignons à savoir Penicillium expansum, Alternaria sp et Résopus stolonifer responsables de la pourriture de la pomme dans les entrepôts de stockage.

\section{Résultats et discussion}

II-1. Synthèse des composés 3a-c [2-(1-méthyl-3-arylprop-2-énylidène)-5méthylcyclohéxanone] :

La synthèse de nouveaux produits a été effectuée par la condensation de la R-(+)pulégone avec les arylaldéhydes en présence de l'hydrure de sodium $(\mathrm{NaH})$ sous l'irradiation micro-onde en milieu sec selon les réactions décrites dans le schéma 1 . Les réactions sont activées par l'exposition à une irradiation aux micro-ondes en milieu sec sous une puissance de $500 \mathrm{w}$ et dans un temps de $12 \mathrm{mn}$. Les résultats obtenus sont regroupés dans le tableau 1.

Tableau 1 : Les rendements et les températures de fusion des produits synthétisés

\begin{tabular}{cccc}
\hline Produit & $\mathrm{R}$ & $\begin{array}{l}\mathrm{Rdt} \\
(\%)\end{array}$ & $\begin{array}{c}\mathrm{Pf} \\
\left({ }^{\circ} \mathrm{C}\right)\end{array}$ \\
\hline \hline $3 \mathrm{a}$ & $\mathrm{C}_{6} \mathrm{H}_{5}$ & 90 & 132 \\
$3 \mathrm{~b}$ & $\mathrm{C}_{6} \mathrm{H}_{4} \mathrm{O}_{3}$ & 85 & 140 \\
$3 \mathrm{c}$ & $\mathrm{C}_{4} \mathrm{H}_{4} \mathrm{O}$ & 83 & 120 \\
\hline
\end{tabular}


Schéma 1 : Condensation des arylaldéhydes avec la R-(+)-pulégone en présence de NaH assisté par micro-ondes en milieu sec.<smiles>CC(C)=C1CCC(C)CC1=O</smiles>

$\underline{1}$<smiles></smiles>

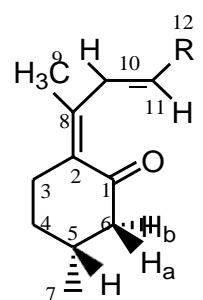

$\underline{\text { 3a-c }}$

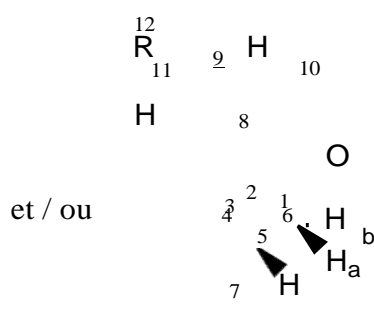

$\underline{\text { 3'a-c }}$ 
Il apparait clairement que les dérivés de la R-(+)-pulégone synthétisés sont obtenus avec de bons rendements, dans des conditions propres sans solvant, économiques et très simples à mettre en œuvre, contrairement au chauffage classique. Ainsi, la technique de four à micro-ondes en milieu sec peut jouer un rôle important dans la synthèse de nouveaux dérivés de la R-(+)-pulégone.

Après purification par chromatographie sur colonne de gel de silice, tous les composés synthétisés 3a-c ont été caractérisés par leurs données spectrales en se basant sur les analyses spectroscopiques IR, RMN du ${ }^{1} \mathrm{H}$ et RMN du ${ }^{13} \mathrm{C}$.

Les spectres IR montrent la présence des bandes d'absorptions des groupements carbonyles. Ces bandes apparaissent respectivement vers 1672,1640 et $1670 \mathrm{~cm}^{-1}$ pour $3 \mathrm{a}, 3 \mathrm{~b}$ et $3 \mathrm{c}$.

Les spectres $\mathrm{RMN}{ }^{1} \mathrm{H}$ et $\mathrm{RMN}{ }^{13} \mathrm{C}$ de tous les composés ont présenté des signaux appropriés avec les structures proposées. En effet, les spectres RMN du ${ }^{1} \mathrm{H}$ des produits $3 \mathrm{a}-\mathrm{c}$ montrent seulement la présence des signaux des deux méthyles et la disparition du signal de l'un des méthyles géminés de la pulégone de départ. Ainsi, la présence des protons aromatiques, montre que la condensation a eu lieu comme elle confirme la formation d'une double liaison $\mathrm{C}=\mathrm{C}$ par la présence d'un système éthylénique avec une constante de couplage de l'ordre de 16,5 Hz, ce qui prouve qu'il s'agit d'une stéréochimie de type trans. Alors que la conservation du reste du squelette de la pulégone indiquant ainsi que l'attaque nucléophile sur le carbone en $\alpha \mathrm{du} C=\mathrm{O}$ n'a pas eu lieu.

Par ailleurs, les spectres de la $\mathrm{RMN}$ du ${ }^{13} \mathrm{C}$ confirment les structures des produits $\underline{\mathbf{3 a}-\mathbf{c}}$ obtenus. En effet, on observe le pic correspondant au groupement carbonyle de l'énone de départ qui apparait à 201,7; 190,5 et 196,0 ppm respectivement ainsi la conservation des signaux du squelette carboné de la pulégone avec ses différentes composantes sauf la disparition du signal de l'un des carbones hybridés $\mathrm{sp}^{3}$ et l'apparition des signaux d'autres carbones hybridés $\mathrm{sp}^{2}$.

Ces réactions induisent l'attaque de l'un des méthyles géminés de la pulégone en formant une double liaison $\mathrm{C}=\mathrm{C}$ avec l'arylaldéhyde et en gardant le reste du squelette de la pulégone. La double liaison $\mathrm{C}=\mathrm{C}$ formée aura un effet sur la réactivité de la molécule en accentuant aussi la polarisation au niveau de l'atome d'oxygène.

Cependant, ces analyses spectroscopiques usuelles, ne peuvent pas mettre en évidence le méthyle géminé attaqué. Mais, il ressort de la littérature, que le groupe méthyle géminé cis ce caractérise par une réactivité plus importante par rapport à l'autre groupe méthyle géminé trans. Cette constatation ce révèle à partir de la réaction d'isomérisation de la pulégone en isopulégone en formant la double liaison avec le méthyle géminé cis du groupement carbonyle [51-52], ainsi, la transformation enzymatique de la pulégone en menthofurane passe par l'hydroxylation de méthyle géminé cis suivie d'une cyclisation intramoléculaire puis déshydratation [53-55]. Quant à la chloration de la pulégone au niveau de méthyle géminé trans, ce réalise en protégeant le méthyle géminé cis [56]. Il résulte de ces études que les protons du méthyle géminé cis ont un pouvoir acide plus important par rapport aux autres protons. De ces considérations, les structures les plus probables pour nos produits seront 3ac. Ces nouveaux systèmes synthétisés nous servent comme éléments de base pour la préparation des hétérocycles.

II-2. Activité antifongique des 2-(1-méthyl-3-arylprop-2-énylidène)-5-méthylcyclohéxanone 3a-c vis-à-vis des trois souches de champignons $P$. expansum. Alternariat $s p$. et $R$. stolonifer :

L'étude de l'activité antifongique des produits synthétisés a été réalisée par la 
méthode de contact direct sur le milieu de culture PDA [57-58]. Cette technique consiste à placer au centre de la boite de Pétri contenant le milieu gélosé et le produit à étudier un disque mycélien de $2 \mathrm{~mm}$ de diamètre. Les cultures sont incubées à $25^{\circ} \mathrm{C}$.

La croissance mycélienne est estimée pendant la durée d'incubation par la mesure chaque jour de la moyenne des deux diamètres perpendiculaires des colonies. Pour chaque produit à tester on utilise trois concentrations différentes $\left(\mathrm{C} 1=10^{-4}, \mathrm{C} 2=10^{-3}\right.$ et $\mathrm{C} 3=10^{-2}$ $\mathrm{mol} / \mathrm{l}$ ), chaque test est répété trois fois.

La révélation de l'efficacité du produit sur le développement d'une souche fongique est réalisée par la mesure du diamètre de la moisissure de la colonie sur le milieu de culture avec et sans produit durant sept jours d'incubation. L'évaluation de l'effet d'inhibition exercé par chaque produit est estimée par le calcul du pourcentage d'inhibition de la croissance mycélienne selon la formule suivante $(\% \mathrm{I})=\left[\left(\mathrm{D}_{1}-\mathrm{D}_{2}\right) / \mathrm{D}_{1}\right] \mathrm{x}$ 100) avec $D_{1}$ : le diamètre de la colonie sur milieu de culture sans produit et $D_{2}$ : le diamètre de la colonie sur milieu de culture avec produit [59] (tableaux 2 et 3).

Tableau 2 : pourcentage d'inhibition d'Alternaria sp.

\begin{tabular}{lccc}
\hline & & Concentration \\
Produit & $10^{-4}$ & $10^{-3}$ & \\
\cline { 2 - 4 } & $\mathrm{mol} / \mathrm{l}$ & $\mathrm{mol} / \mathrm{l}$ & $\mathrm{mol}$ \\
\hline$\underline{\mathbf{3 a}}$ & 67.80 & 68.87 & $\mathrm{~mol} / \mathrm{l}$ \\
$\underline{\mathbf{3 b}}$ & 71.63 & 76.10 & 84.40 \\
$\underline{\mathbf{3 c}}$ & 71.67 & 81.10 & 86.67
\end{tabular}

Tableau 3 : pourcentage d'inhibition de $P$. expansum

\begin{tabular}{lccc} 
Produit & \multicolumn{3}{c}{} \\
\cline { 2 - 4 } & $10^{-4}$ & $10^{-5}$ & $10^{-4}$ \\
$\mathrm{~mol} / \mathrm{l}$ & $\mathrm{mol} / \mathrm{l}$ & $\mathrm{mol} / \mathrm{l}$ \\
\hline$\underline{\mathbf{3 a}}$ & 78.87 & 80.27 & 82.23 \\
$\underline{\mathbf{3 b}}$ & 80.53 & 82.76 & 87.23 \\
$\underline{\mathbf{3 c}}$ & 86.10 & 87.30 & 88.80
\end{tabular}

Ces dérivés terpéniques oxygénés présentent des activités antifongiques contre $P$. expansum. Alternariat $s p$. dont le pourcentage d'inhibition croit en fonction de la concentration et qui varie d'un produit à un autre. Les données de la littérature indiquent que l'activité des terpènes oxygénés vis-à-vis des pathogènes fongiques est due à leurs interfaces avec les réactions enzymatiques lors de la synthèse de la paroi cellulaire 
induisant des altérations de la perméabilité cellulaire en perturbant la structure des lipides et entraînant ainsi des changements de propriétés des fonctions membranaires [60-61]. Il semble que la présence des groupements donneurs d'électrons favorisant la motivation électronique au niveau du groupement carbonyle, sont à l'origine de cette variation d'efficacité. En effet, le remplacement d'un hydrogène en position para par un groupement méthoxy (produit $\underline{\mathbf{3 b}}$ ) ou la substitution du noyau phényle par un noyau furanique (produit $\underline{\mathbf{3 c}}$ ), fait augmenter légèrement l'activité inhibitrice.

Cependant la souche de champignon $R$. stolonifer exprime une résistance aux produits 3a-c. Ce comportement peut être dû au fait que ce champignon neutralise l'effet des concentrations testées par son métabolisme normal ou la dose appliquée pourrait être insuffisante pour permettre au produit de réagir face au pathogène. Mais, une différence qualitative est observée entre la souche vierge et la souche traitée. Dans le cas de la première, le tapis mycélien reste noir, alors que le tapis mycélien de la souche traité est blanc. Cela signifie que la sporulation est altérée ou inhibée par le traitement. Bien que ces produits soient inefficaces à faible concentration vis-à-vis de la croissance mycélienne de $R$. stolonifer, elles présentent un effet sur les sporocystes (figure 1).

\section{III.Partie expérimentale}

\section{III-1. Généralités}

Les points de fusion ont été mesurés sur banc de kofler. Les spectres de $\mathrm{RMN}{ }^{1} \mathrm{H}$ et ${ }^{13} \mathrm{C}$ ont été enregistrés sur un appareil Bruker (300Mhz pour le $\mathrm{RMH}{ }^{1} \mathrm{H}$ et $75 \mathrm{Mhz}$ pour RMN ${ }^{13} \mathrm{C}$ ). Les spectres IR ont été réalisés sur un spectromètre FT/ IR-4100 Type A. Toutes les réactions ont été contrôlées par chromatographie sur couche mince en silice (CCM gel de silice $\left.60 \mathrm{~F}_{254}\right)$

\section{Figure 1 :}

a) Les trois souches de champignons isolés de la pomme pourrie en conservation

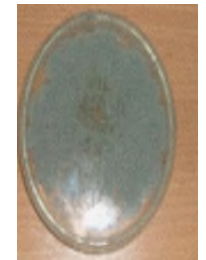

Souche pure de Penicillium expansum

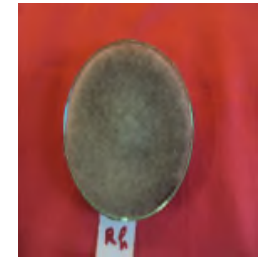

Souche pure de Rhizopus stolonifer

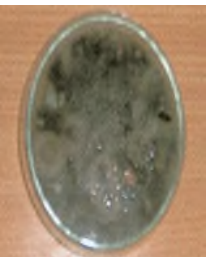

Souche pure d'Alternaria $s p$

b) Effet inhibiteur du produit [2-(1-méthyl-3-phénylprop-2-énylidène)-5-
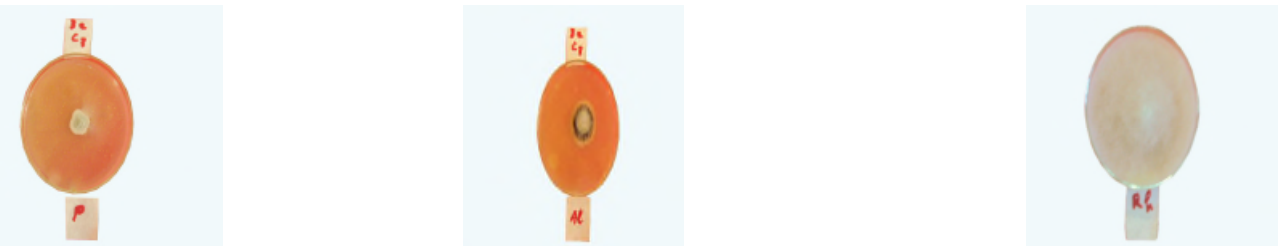

méthylcyclohexanone] à concentration $10^{-2} \mathrm{~mol} / \mathrm{l} \underline{\mathbf{3 a}}$ sur les trois souches de champignons 
c) Effet inhibiteur du produit [2-(1-méthyl-3-p-méthoxyphénylprop-2-énylidène)-5méthylcyclohexanone] à concentration $10^{-2} \mathrm{~mol} / \mathrm{l} \underline{\mathbf{3 b}}$ sur les trois souches de champignons
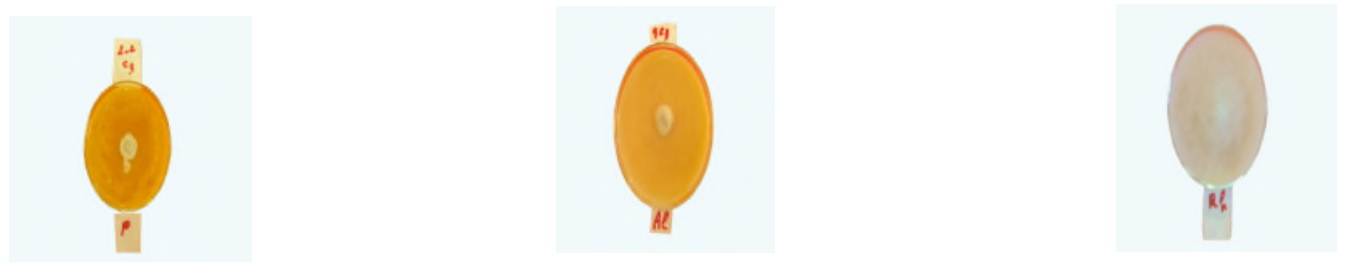

d) Effet inhibiteur du produit [2-(1-méthyl-3-furanylprop-2-énylidène)-5méthylcyclohexanone] à concentration $10^{-2} \mathrm{~mol} / \mathrm{l} \underline{\mathbf{3 c}}$ sur les trois souches de champignons
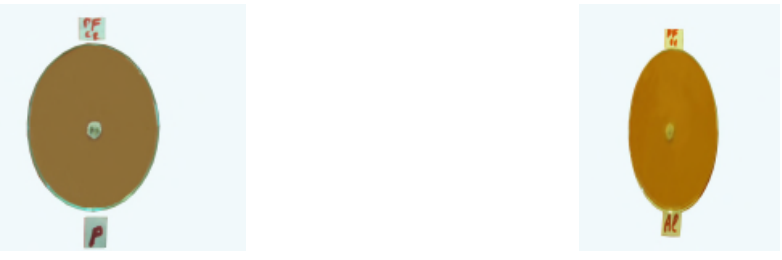

\section{III-2. Synthèse des composés $\underline{3 a-c}$}

Dans un erlenmeyer de $250 \mathrm{ml}$ on mélange $3 \mathrm{~g}(0,02 \mathrm{~mol})$ de la R-(+)-pulégonet $\underline{\mathbf{1}}$, 2,1 $\mathrm{g}(0,02 \mathrm{~mol})$ de l'aryaldéhyde $\underline{\mathbf{2}}, 0,47 \mathrm{~g}$ d'hydrure de sodium et $5 \mathrm{~g}$ de la silice $\left(\mathrm{SiO}_{2}\right)$. Le mélange est activé par l'irradiation micro-ondes sous une puissance de $500 \mathrm{w}$ pendant $12 \mathrm{mn}$. Les produits synthétisés sont extraits du support minéral avec du dichlorométhane. Après élimination $\mathrm{du}$ solvant sous pression réduite, les produits sont purifiés par chromatographique sur colonne de gel de silice (éluant : hexane / dichlorométhane : 80 / 20).

III-2-1. Identification du Produit 2-(1-méthyl-3-phénylprop-2-énylidène)-5méthylcyclohéxanone $\underline{\mathbf{3 a}}$

$\mathrm{Rdt}=90 \%, \mathrm{Pf}=132{ }^{\circ} \mathrm{C}$, Couleur : rouge brique.

$\mathrm{RMN}{ }^{1} \mathrm{H}\left(300 \mathrm{MHz}, \mathrm{CDCl}_{3}\right), \delta\left(\right.$ en ppm), J(Hz) : $0.9\left(\mathrm{~m}, 1 \mathrm{H},{ }^{5} \mathrm{CH}\right) ; 1.1\left(\mathrm{~d}, 3 \mathrm{H},{ }^{7} \mathrm{CH}_{3}\right.$, $\mathrm{J}=6.2 \mathrm{~Hz}) ; 1.35\left(\mathrm{~m}, 2 \mathrm{H},{ }^{4} \mathrm{CH}_{2}\right) ; 1.67\left(\mathrm{~s}, 3 \mathrm{H},{ }^{9} \mathrm{CH}_{3}\right) ; 2.4\left(\mathrm{~m}, 2 \mathrm{H},{ }^{3} \mathrm{CH}_{2}\right) ; 2.5\left(\mathrm{dd}, 1 \mathrm{H}, \mathrm{H}_{\mathrm{a}}^{6}\right.$, $\left.\mathrm{J}\left(\mathrm{H}_{\mathrm{a}}^{6}-\mathrm{H}_{\mathrm{b}}^{6}\right)=16 \mathrm{~Hz}, \mathrm{~J}\left(\mathrm{H}_{\mathrm{a}}^{6}-\mathrm{H}^{5}\right)=7.5 \mathrm{~Hz}\right) ; 2.8\left(\mathrm{dd}, 1 \mathrm{H}, \mathrm{H}_{\mathrm{b}}^{6}, \mathrm{~J}\left(\mathrm{H}_{\mathrm{b}}^{6}-\mathrm{H}_{\mathrm{a}}^{6}\right)=16 \mathrm{~Hz}\right.$, $\left.\mathrm{J}\left(\mathrm{H}_{\mathrm{b}}^{6}-\mathrm{H}^{5}\right)=4.5 \mathrm{~Hz}\right) ; 6.7\left(\mathrm{~d}, 1 \mathrm{H},{ }^{11} \mathrm{CH}, \mathrm{J}\left(\mathrm{H}^{11}-\mathrm{H}^{10}\right)=16.5 \mathrm{~Hz}\right) ; \mathrm{m} 7.15-7.5(5 \mathrm{H}, \mathrm{H}$ aromatiques); $7.65\left(\mathrm{~d}, 1 \mathrm{H},{ }^{10} \mathrm{CH}, \mathrm{J}\left(\mathrm{H}^{10}-\mathrm{H}^{11}\right)=16.5 \mathrm{~Hz}\right)$.

$\mathrm{RMN}{ }^{13} \mathrm{C}\left(75 \mathrm{MHz}, \mathrm{CDCl}_{3}\right) \delta(\mathrm{en} \mathrm{ppm}): 14.8-21.8-30.6-31.45-31.6-51.02$

(Csaturés); 127.0 - 127.6 - 128.5 - 128.8 - 129.0 - 130.7 - 131.8 - 135.4 - 137.6 - 138.2

(C aromatiques et vinyliques); $201.7\left(\mathrm{C}^{1}\right)$.

IR $(\mathrm{KBr}),\left(v\right.$ en $\left.\mathrm{cm}^{-1}\right): 3059 ; 3025 ; 2952 ; 1672 ; 1651 ; 1601 ; 754 ; 695$.

III-2-2. Identification du Produit 2-(1-méthyl-3-p-méthoxyphénylprop-2énylidène)-5- méthylcyclohéxanone $\underline{3 \mathrm{~b}}$ :

$\mathrm{Rdt}=85 \%, \mathrm{Pf}=140^{\circ} \mathrm{C}$, Couleur : Jaune claire. 
$\mathrm{RMN}^{1} \mathrm{H}\left(300 \mathrm{MHz}, \mathrm{CDCl}_{3}\right), \delta\left(\right.$ en ppm), J(Hz) : $0.95\left(\mathrm{~m}, 1 \mathrm{H},{ }_{\mathrm{b}}^{5} \mathrm{CH}\right) ; 0.9\left(\mathrm{~d}, 3 \mathrm{H},{ }^{7} \mathrm{CH}_{3}\right.$, $\mathrm{J}=6.2 \mathrm{~Hz}) ; 1.25\left(\mathrm{~m}, 2 \mathrm{H},{ }^{4} \mathrm{CH}_{2}\right) ; 1.87\left(\mathrm{~m}, 2 \mathrm{H},{ }^{3} \mathrm{CH}_{2}\right) ; 1.87\left(\mathrm{~s}, 3 \mathrm{H},{ }^{9} \mathrm{CH}_{3}\right) ; 2.35\left(\mathrm{dd}, 1 \mathrm{H}, \mathrm{H}_{\mathrm{a}}^{6}\right.$, $\left.\mathrm{J}\left(\mathrm{H}_{\mathrm{a}}^{6}-\mathrm{H}^{6}{ }_{\mathrm{b}}\right)=13 \mathrm{~Hz}, \mathrm{~J}\left(\mathrm{H}^{6}-\mathrm{H}^{5}\right)=7.5 \mathrm{~Hz}\right) ; 2.55\left(\mathrm{dd}, 1 \mathrm{H}, \mathrm{H}^{6}, \mathrm{~J}\left(\mathrm{H}^{6}-\mathrm{H}^{6}\right)=13 \mathrm{~Hz}\right.$, $\left.\mathrm{J}\left(\mathrm{H}^{6}-\mathrm{H}^{5}\right)=4.5 \mathrm{~Hz}\right) ; 3.65\left(\mathrm{~s}, 3 \mathrm{H}, \mathrm{OCH}_{3}\right) ; 6.6-6.8(\mathrm{~m}, 4 \mathrm{H}$ aromatiques $) ; 6.95\left(\mathrm{~d}, 1 \mathrm{H},{ }^{11} \mathrm{CH}\right.$, $\mathrm{J}=16.5 \mathrm{~Hz}) ; 7.55\left(\mathrm{~d}, 1 \mathrm{H},{ }^{10} \mathrm{CH}, \mathrm{J}=16.5 \mathrm{~Hz}\right)$.

$\mathrm{RMN}{ }^{13} \mathrm{C}\left(75 \mathrm{MHz}, \mathrm{CDCl}_{3}\right) \delta($ en ppm) : 22.0-28.5-29.6-31.4-32.7-50.7 - 55.1

(Csaturés); 114.1 - 124.9 - 125.15 - 127.0 - 131.7 - 131.8 - 139.4 - 139.6 - 141.8 -

159.5 (Caromatiques et vinyliques); $190.5\left(\mathrm{C}^{1}\right)$.

IR $(\mathrm{KBr}),\left(v \mathrm{en} \mathrm{cm}^{-1}\right): 3030 ; 2923 ; 1640 ; 730$.

III-2-3. Identification du Produit 2-(1-méthyl-3-furanylprop-2-énylidène)-5-

méthylcyclohéxanone $\underline{3 c}$

$\mathrm{Rdt}=83 \%, \mathrm{Pf}=120^{\circ} \mathrm{C}$, Couleur : Jaune claire.

RMN $1 \mathrm{H}\left(300 \mathrm{MHz}, \mathrm{CDCl}_{3}\right), \delta$ (en ppm), J(Hz) : $0.8\left(\mathrm{~m}, 1 \mathrm{H},{ }^{5} \mathrm{CH}\right) ; 0.85\left(\mathrm{~d}, 3 \mathrm{H},{ }^{7} \mathrm{CH}_{3}, \mathrm{~J}=6.2\right.$ $\mathrm{Hz}) ; 1.2\left(\mathrm{~m}, 1 \mathrm{H},{ }^{3} \mathrm{CH}_{2}\right) ; 1.9\left(\mathrm{~s}, 3 \mathrm{H},{ }^{9} \mathrm{CH}_{3}\right) ; 2.1_{\mathrm{b}}\left(\mathrm{m}, \underset{\mathrm{a}}{2 \mathrm{H}},{ }^{4} \mathrm{CH}_{2}\right) ; 2.4\left(\mathrm{dd}, 1 \mathrm{H}, \mathrm{H}_{\mathrm{b}}^{6}{ }_{\mathrm{a}}\right.$, $\left.\left.\mathrm{J}\left(\mathrm{H}_{\mathrm{a}}^{6}-\mathrm{H}^{6}\right)^{\mathrm{a}}\right)=13 \mathrm{~Hz}, \mathrm{~J}\left(\mathrm{H}^{6}-\mathrm{H}^{5}\right)=7.5 \mathrm{~Hz}\right) ; 2.8\left(\mathrm{dd}, 1 \mathrm{H}, \mathrm{H}^{6}, \mathrm{~J}\left(\mathrm{H}^{6}-\mathrm{H}^{6}\right)=13 \mathrm{~Hz}\right.$, $\left.\mathrm{J}\left(\mathrm{H}^{6}-\mathrm{H}^{5}\right)=4.5 \mathrm{~Hz}\right), 6.45\left(\mathrm{~d}, 1 \mathrm{H},{ }^{11} \mathrm{CH}, \mathrm{J}=16.3 \mathrm{HZ}\right) ; 6.65\left(\mathrm{~d}, 1 \mathrm{H},{ }^{10} \mathrm{CH}, \mathrm{J}=16.3 \mathrm{~Hz}\right)$; 6.2 - 7.55 (m, 3H, Haromatiques).

$\mathrm{RMN}{ }^{13} \mathrm{C}\left(75 \mathrm{MHz}, \mathrm{CDCl}_{3}\right) \delta($ en ppm $):(11.42-18.75-22.62-29.05-29.69-58.48(\mathrm{C}$ saturés) ; 112.47 - $114.84-115.82$ - 133.07 - 145.32 - $150.65-152.0-172.43$

(Caromatiques et vinyliques); $196.0\left(\mathrm{C}^{1}\right)$. IR (KBr), $\left(v\right.$ en $\left.\mathrm{cm}^{-1}\right): 3030 ; 2928 ; 1670$.

\section{III-3. Préparation de l'agent pathogène}

Les isolats de $P$. Expansum, l'Alternariat $S p$. et $R$. Stolonifer dans cette étude ont été obtenus à partir des plants des pommes présentant des pourritures. Ces derniers sont prélevés des entrepôts de stockage. L'identification des espèces fongiques est basée sur plusieurs clés de détermination [62- 65].

\section{IV.Conclusion}

La méthode d'activation sous irradiation micro-ondes décrit dans ce travail ouvre des perspectives intéressantes dans la valorisation des substances naturelles. Elle présente une certaine simplicité au niveau du protocole opératoire comme elle révèle une intéressante potentialité respectueuse de l'environnement dans la mesure où elle fait intervenir des milieux propres sans solvant. En effet, il nous a été possible à l'aide de cette approche synthétique de préparer de nouveaux dérivés de la R-(+)-pulégone avec des rendements remarquables et dans un temps très court. Les produits synthétisés sont susceptibles d'être des précurseurs des produits à valeurs ajoutées.

À l'issue de cette étude biologique, ces nouveaux produits synthétisés ont un effet inhibiteur significatif sur la croissance mycélienne des deux champignons $P$. expansum. Alternariat sp. Par contre la souche de champignon $R$. stolonifer manifeste une résistance 
envers cette série de produit, mais avec l'absence de la couleur brune donnée par les sporocystes.

\section{Références}

[1] Sezik E., Tumen G., Kho B., J. Flavour Fragrance, 6, 1991, 101-103.

[2] Meral G. E., Konyalioglu S., Ozturk B., Fitoterapia, 73, 2002, 716718.

[3] Ravid U., J. Flavour and Frangrance, 9, 1994, 205-207.

[4] Soulerles C., Katsiotis S., Plant. Med. Pytother, 22, 1988, 180.

[5] Madyastha. K. M., Paul Raj. C., Biochem, Biophys. Res. Commun, 297, 2002, 202-

205. [6] Bclaut. J. L. I., Ichhes. S. K., Tysou. L. A., Seamans. T. W., Int. J. Pest.

Management, 107(4), 1997, 303.

[7] Hall. R. A., Oser. B. L., Food Technology, 19, 1965, 253-271.

[8] Tepe B., Sokmen M., Sokmen A., Daferera D., Polissiou M., J. of Food Engineering, 69, 2005, 335-342.

[9] Mata A. T., Proença C., Ferreira A. R., Serralheiro M. L. M., Nogueira J. M. F., Araujo M. E. M., Food Chemistry, 103, 2007, 778-786.

[10] Hanamanthagouda M. S., Kakkalameli S. B., Naik P. M., Nagella P., Seetharamareddy

H., Murthy H. N., Food Chemistry, 118, 2010, 836-839.

[11] Romero-Jiménez M., Campos-Sanchez J., Amalla M., Munoz-Serrano A., Alonso- Moraga A., Mutat. Res., 585, 2005, 147-155.

[12] Naeini A., Khosravi A. R., Chitsaz M., Shokri H., Kamlnejad M., J. Mycologie Médicale, 19, 2009, 168-172.

[13] Bakkalu F., Averbeck S., Averbeck D., Idaomar M., Food Chemical Toxicology, 46 (2), 2008, 446-475.

[14] Asekun O. T., Grierson D. S., Afolayan A. J., Food Chemistry, 101, 2007, $995-$

998. [15] Tisserand R., ISBN: 0-7225-2645-8, 1993.

[16] Van Wyk B. E., Gericke N., People's plants. Pretoria: Briza Publications, 128, 2000, 324-327.

[17] Phillips R., Foy N., ISBN: 0-330-30725-8, 1990.

[18] Zeichen de Sa, R., Rey A., Arganaraz E., Bindstein E., J. Ethnopharmacol, 69, 2000, 93- 98.

[19] Mimica D. N., Bozin B., Sokovic M., Mihailovic B., Matavulj M., Planta Medica., 69 (5), 2003, 413-419.

[20] Umezu T., Pharmacology Biochemistry and Behavior, 94, 2010, 497-502.

[21] Petrakis E. A., Kimbaris A. C., Pappas C. S., Tarantilis P. A., Polissiou M. G. J., J. Agric. Food Chem., 57, 2009, 10044-10048.

[22] Souza A. A. S., Soares, P. M. G., Almeida, A. N. S., Maia, A. R., Souza, E. P., J. of Ethnopharmacology, 130 (2), 2010, 433-436.

[23] Brankovic S. V., Kitic D. V., Radenkovic M. M., Veljkovic S. M., Golubovic T. D., Gen. Physiol. Biophys., 28, 2009, 174-178.

[24] Riahi L., Elferchichi M., Ghazghazi H., Jebali J., Ziadi S., Aouadhi Ch., Chograni H., Zaouali Y., Zoghlami N., Mliki A., Industrial Crops and Products, 49, 2013, 883889.

[25] Rossi Y. E., Canavoso L., Palacios S. M., Fitoterapia, 83, 2012, 336-342.

[26] Zekri N., Amalich S., Boughdad A., Alaoui El Belghiti M., Zair T., Med.

$\mathrm{J}$.

Chemistry, 2 (4), 2013, 607-619.

[27] Lipshutz B. H., Wilhelm R. S., J. Kozlowski, Tetrahedron Lett., 23, 1982, 3755-3758. 
[28] Dauben W. G., Hart D. J., Ipatschi J., Kozikowski A. P., Tetrahedron lett., 1973, 4425-4428.

[29] Ito Y., Kato H., Imari H., Saegusa T., J. Am. Chem. Soc., 84, 1962, 3782.

[30] Bensel N., Hohn J., Marschall H., Weyerstahl P., Chem. Ber., 112, 1979, 2256-

2277. [31] Ghozland F., Maroni-barnaud Y., Maroni P., Bull. Soc. Chim., Fr. N 1-2,

$1974,147$.

[32] Viswanatha V., Rao G. S. K., Indian J. Chem., 13, 1975, 101.

[33] Nerdel F., Dahl H., Justus Liebigs Ann. Chem., 710, 1967, 36-58.

[34] Ohloff G., Osiecki J., Djerassi C., Chem. Ber., 95, 1962, 1400.

[35] Kon G. A. R., Nutland J. H., J. Chem. Soc., 1926, 3101.

[36] Grignard V., Chem. Zentr., 72, 1901, 602-603.

[37] Wolinsky J., Chan D., J. Am. Chem. Soc., 85, 1963, 937-940.

[38] Santelli M., Bertrand M., Bull. Soc. Chim. Fr., 1973, 2326-2331.

[39] Proszenyak M. A., Braendvang M., Chornock C., Lotte Gandenrsen L., Tetrahedron, 65, 2009, 194-199.

[40] Cossy J., Bouzbouz S., Hakiki A., Tetrahedron Letters, $38 \mathrm{~N}^{\circ}$ 51, 1997, 8853-8854.

[41] Brémond P., Vanthuyne N., Audran G., Tetrahedron Letters, 50, 2009, 5723-5725.

[42] Frideling A., Fauve R., Gally J. P., Kenz A., Alkorta I., Elguero J., European Journal of Médicinal Chemistry, 39, 2004, 37-48.

[43] Denmark S. E., Venkatraman S., J. Org. Chem., 71 N 4, 2006, 1668-1676.

[44] Bellioua A., Majidi L., Fhi R., Boudina A., Baouid A., Phys. Chem. News, 33, 2007, 138.

[45] Faska Z., Bellioua A., Bouklah M., Majidi L., Fihi R., Bouyanzer A., Hammouti B., Monatsh Chem., 139, 2008, 1417-1422.

[46] Znini M., Bouklah M., Majidi L., Kharchouf S., Aouniti A., Bouyanzer A., Hammouti B., Costa J., Al Deyab S. S., Int. J. Electrochem. Sci., 6, 2011, 691-704.

[47] Oubair A., Fihi R., Majidi L., Azrour M., and Daran J. C., Acta Crys., E66, 2010, o2391o2392.

[48] Kharchouf S., Majidi L., Abraoui H., Faska Z., Cheminform, Vol 39 N 33, 2008,

[49] Rajender S. Varma, Green Chemistry, 1, 1999, 43-55.

[50] Bougrin K., Loupy A., Soufiaoui M., J. Photochemistry and Photobiologie. Vol 6 (2-3), 2005, 139-167.

[51] Madayastha K. M., Thulasiram H. V., J. Agric. Food Chem., 47, 1999, 1203-1207.

[52] Alonso W R., Rajaonarivony J. I. M., Gershenzon J., Croteau R., Journal of Biological Chemistry, Vol $267 \mathrm{~N}^{\circ} 11,1992,1582-1587$.

[53] Bertea C. M., Schalk M., Karp F., Maffei M., Croteau R., Archives of Biochemistry and Biophysics, Vol $390 \mathrm{~N}^{\circ}$ 2, 2001, 279-286.

[54] Zhou S., Koh H. L., Gao Y., Gong Z. Y., Lee E. J. D., Life Sciences, 74, 2004, 935-968.

[55] Madyastha K. M., Paul Raj C., Biochemical and Biophysical Research Communications, 297, 2002, 202-205.

[56] Hegde Shridhar G., Beckwith D., Doti R., Wolinsky J., J. Org. Chem., V 50 N 6, 1984, 894.

[57] Michra A. K., Dubey N. K., Applied and Environmental Microbiology, 60 (4), 1994, 1101-1105.

[58] Belghazi L., Lahlou N., Alaoui Ismaili M., Abousaouiria T., Habti N., Tantaoui Iraki A., Talbi M., Blaghen M., Fellat-Zarrouck K., Biochimie et Santé, 2002, 38-40.

[59] Hmouni A., Hajlaoui MR., Mlaiki A., OEPP/EPPO Bull., 26, 1996, 697-705.

[60] Inouye S., Tsuruoka T., Watanab M., Takeo K., Akao M., Nishiyama Y., Yamaguchi H., PubMed PMID, 43, 2000, 17-23.

[61] Zambonelli Z, Zechini D’Aulerio A., Blanchi A., Albasini A., J. Phytopatol., 144, 1996, 
491-494.

[62] Barnet H. L., Hunter B. B., Burgess Pub. Com., 1972, 241-254.

[63] Botton B., Breton A., Fevre M., Gauthier S., Guy P., Larpent J. P., Reymond P., Sarglier J. J., Vayssier Y., 2ème édition Collection Biotechnologies, Masson, 1990, 512.

[64] Pitt J. I., Commonwealth Scientific and Industrial Research Organization, Division of Food, 1988, 187.

[65] Samson R. A., Hoekstra E. S., Van C. A. N., Orschot. O., $2^{\text {nd }}$ edition. Centraal bureau Voor Schimmel cultures, 1984, 248. 